\title{
Social-Aware Collaborative Caching Based on User Preferences for D2D Content Sharing
}

\author{
Can Zhang ${ }^{1}$, Dan $\mathrm{Wu}^{1}{ }^{*}$, Liang Ao ${ }^{1}$, Meng Wang ${ }^{1}$, and Yueming Cai ${ }^{1}$ \\ ${ }^{1}$ Army Engineering University of PLA \\ Nanjing 210007, Jiangsu - P. R. China \\ [e-mail: xiliecan@163.com] \\ *Corresponding author: Dan Wu
}

Received May 10, 2019; revised October 28, 2019; accepted December 26, 2019; published March 31, 2020

\begin{abstract}
With rapid growth of content demands, device-to-device (D2D) content sharing is exploited to effectively improve the service quality of users. Considering the limited storage space and various content demands of users, caching schemes are significant. However, most of them ignore the influence of the asynchronous content reuse and the selfishness of users. In this work, the user preferences are defined by exploiting the user-oriented content popularity and the current caching situation, and further, we propose the social-aware rate, which comprehensively reflects the achievable contents download rate affected by the social ties, the caching indicators, and the user preferences. Guided by this, we model the collaborative caching problem by making a trade-off between the redundancy of caching contents and the cache hit ratio, with the goal of maximizing the sum of social-aware rate over the constraint of limited storage space. Due to its intractability, it is computationally reduced to the maximization of a monotone submodular function, subject to a matroid constraint. Subsequently, two social-aware collaborative caching algorithms are designed by leveraging the standard and continuous greedy algorithms respectively, which are proved to achieve different approximation ratios in unequal polynomial-time. We present the simulation results to illustrate the performance of our schemes.
\end{abstract}

Keywords: D2D content sharing, user preferences, social-aware collaborative caching, submodular function, greedy algorithm

A preliminary version of this paper appeared in IEEE ICCT 2017, October 27-30, Chengdu, China. This research was supported by the Jiangsu Provincial Natural Science Foundation for Excellent Young Scholars (No. BK20170089) and the National Natural Science Foundation of China (No. 61671474, No. 61771487). 


\section{Introduction}

W ith the dramatical increase of content demands, wireless content sharing is experiencing the proliferation of data traffic [1]. In a traditional cellular network, as the center of service system, the base stations (BS) will undertake the scheduling of massive access devices and information, leading to the rapid rise of wireless resource consumption. Simultaneously, it is difficult for the BS to meet the content demands of all the users. In fact, there are a large number of mobile devices in the network, whose data storage, computing processing and information interaction capability can be regarded as available resources. Then, making full use of these widely distributed mobile devices is one of the effective ways to relieve the pressure of BS and overcome the shortage of wireless resources. Based on this, D2D communication technology is adopted, which allows adjacent devices communicate directly with each other [2]-[4]. It can not only relieve the pressure of BS and improve spectrum efficiency, but also shorten communication delay. In this way, based on the proximity and data pass-through features of D2D communications, widely distributed mobile devices can be employed to resolve the problem for massive content sharing. In this regards, device-to-device (D2D) content sharing is proposed, which takes advantage of D2D communications by addressing the issues about the content preset, matching and transmission [5], [6].

In a D2D content sharing scenario, the contents are cached in multiple mobile devices, and these devices can share contents with each other by establishing D2D links, which can improve the quality of service of users, such as reducing the download delay, increasing the download rate, and so on. The devices are divided into two types, i.e., the content demanders (CD) who request their desired contents, and the potential content providers (CP) who can selectively cache some contents in their limited storage space to meet some CDs' content demands. In this regard, if a $\mathrm{CP}$ receives a request from a $\mathrm{CD}$ and it just has this content, it may send this content to the latter via D2D communication. The authors in [7] provide an overview on D2D content sharing, and point out that the D2D content sharing approach can achieve the near-optimal throughput scaling law, in comparison with the traditional approaches that the contents are obtained from the BS based on cellular communications.

However, considering the limited storage space of the CPs and various demands of the CDs, the CPs cannot cache all contents that may be requested by the CDs, and the contents cached in the CPs cannot meet all the content demands of CDs. In fact, the majority of content requests from the CDs focus on the minority of popular contents, while a large number of contents remain unpopular. Therefore, the CPs cannot cache contents at random. It is desired that the cached contents are most likely to be requested by the CDs in the near future. Hence, how to selectively cache contents is very important to D2D content sharing [8]. The content caching is a key question for D2D content sharing, which can leverage the limited storage space of the CPs to meet more content demands of the CDs.

Indeed, there exist many works to focus on the design of caching schemes in D2D content sharing scenarios. In particular, most of them evaluate the performance of caching schemes from the two different perspectives, i.e., the caching gain and the caching cost. Specifically, the former mainly involves the network throughput in [9] and the cache hit ratio in [10]. The latter mainly involves the energy consumption in [11] and the delay in [12]. In either case, the influence of the asynchronous content reuse is ignored. That is, a few popular contents account for a large part of the traffic, however, they are requested by the CDs at different times. In this context, if some contents are cached too many times, the storage space of CPs may be wasted; otherwise, the cache hit ratio of the CDs may be reduced. Hence, how to make a trade-off 
between the redundancy of caching contents and the cache hit ratio of the CDs is significant for designing the caching schemes. Most of these existing approaches only consider the physical layer information, and have a common assumption that the users are altruistic. In fact, considering the consumption of storage space and energy, the CPs may not be willing to cache and share contents requested by the CDs. Hence, how to incentivize the CPs to cache contents is also the key problem. As such, the following two questions should be answered.

Question 1: How to design the caching scheme with respect to the asynchronous content reuse to strike a balance between the redundancy of caching contents and the cache hit ratio?

In the design of caching schemes, the content popularity is generally exploited to improve the availability of preset contents, such as increasing the cache hit ratio of the CDs, e.g., [7],[13]-[17]. In essence, the content popularity characterizes the probability of request for every content from all CDs [7]. It is usually regarded as the Zipf distribution, so as to show that the majority of requests from the CDs focus on the minority of popular contents. This content popularity implies that the content demands of CDs are identical, and it just enables to show the collective preferences from all the CDs [14]. Accordingly, it is hard to reflect the diversity of content demands of individual CDs, which cannot conform to the fact that the content demands of different CDs may well be diverse. To this end, one of the most effective ways to increase the cache hit ratio is to amend the existing content popularity by adding the differences of individual users. For instance, the authors in [16] propose the request model for correlated locations, and the content demands of CDs is modeled as the conditional probability model based on the correlation value between users and contents in [17]. Inspired by these, we propose the user-oriented content popularity to enhance the cache hit ratio, which characterizes the content demands of different CDs.

However, the above methods for improving the content popularity may result in the redundancy of caching contents and the waste of storage space for the asynchronous content reuse, that is, the same contents are cached too many times, especially the popular contents. As such, the collaborative caching is needed, which can be exploited to make a tradeoff between the redundancy of caching contents and the cache hit ratio. The core of collaborative caching model is that the CPs interact with each other to cache selectively contents, so that they can cache more diversified contents. For instance, the authors in [18] discuss the cooperation among the BSs and remote antenna units based on rateless network coding in distributed MIMO systems. The authors in [19] propose to divide users and contents into several sections, and then, the CPs in every section cache different contents so that they can provide more kinds of contents. In addition, the interaction between the caching situation of CPs is exploited in [8], and then, the collaborative caching is achieved by exploiting the content popularity and the current caching proportion of different contents. Note that, the excessive amendment may make the rank of popularity of all the contents be out of action. For example, once the most popular content is cached, its rank of content popularity becomes the lowest, and it will be only cached in a CP. However, the most popular content may be requested by many CDs simultaneously. Inspired by this, we try to achieve the collaborative caching by adding the user preferences, which is designed to characterizes the caching value of the contents for the CDs, based on the user-oriented content popularity and the current neighbouring caching situation.

Question 2: How to incentivize the CPs to cache and share contents requested by the CDs?

Considering the consumption of storage space and energy, the CPs with the selfish nature may not be willing to cache contents requested by the CDs. Hence, the design of caching incentive mechanism is significant in D2D content sharing scenarios, such as the monetary incentive in [20]. However, the monetary incentive mechanism ignores the influence of the social relationships among users. In essence, the CPs may be willing to share contents with the 
CDs who have stronger social relationships with them. In order to create incentives for caching, the social information can be exploited [21]. For instance, the authors in [22] propose a hypergraph framework to design the content caching by considering the social ties among users. In [23], the social information and the physical information is jointly adopted to obtain higher performance. However, these existing works only combine the physical layer information and the social information between the CPs and the CDs to stimulate the CPs to share contents, and ignore the influence of social information among the CDs. In fact, the social relationships among the CDs can be also introduced to incentive the CPs to cache contents. Because the social ties among the CDs can reflect their interest similarity, and characterize the correlation of content demands of the CDs, so that we can exploit them to properly characterize different content demands of CDs. This will be helpful to select the contents most likely requested by the CDs in the near future, and incentive the CPs to cache these valuable contents.

Motivated by these observations, we consider the social-aware collaborative caching based on the user preferences in D2D content sharing scenarios. The main contributions are summarized as follows:

i) The user preferences are defined to characterize the caching values of the contents for different CDs, which are composed of the user-oriented content popularity and the current caching situation. In particular, the former is proposed by considering both the content popularity and social ties, so as to characterize various content demands of individual users.

ii) The social-aware rate is proposed, which characterizes the achievable download rate affected by the social ties, the caching indicators, and the user preferences. With the goal of maximizing the sum of social-aware rate, the social-aware collaborative caching problem is modeled to strike a balance between the redundancy of caching contents and the cache hit ratio.

iii) The problem is simplified to be the maximization of a monotone submodular function over a matroid constraint. Then, two kinds of social-aware collaborative caching algorithms are designed by leveraging the standard and continuous greedy algorithms respectively, which are proved to achieve different approximation ratios in different polynomial-time.

The remainder of this paper is organized as follows: In Section 2 and 3, we describe the system model and problem formulation. In Section 4, the above problem is reduced to be the maximization of a monotone submodular function over a matroid constraint. Then our algorithms are designed and analyzed in Section 5. Finally, some simulations are done to prove the performance of the algorithms in Section 6 before the conclusion in Section 7.

\section{System Model}

As shown in Fig. 1, there is a central base station (BS) and multiple users in a cell. Some users can cache some contents in their limited storage space, and some users want to acquire some contents. Hence, the users are divided into two types, i.e., the CPs who can cache some contents to meet others' content demands and do temporally not retrieve contents for themselves, and the $\mathrm{CDs}$ who request their desired contents. Then, they form $\mathrm{P}=\left\{p_{1}, \mathrm{~L}, p_{j}, \mathrm{~L}, p_{N}\right\}$ and $\mathrm{D}=\left\{d_{1}, \mathrm{~L}, d_{i}, \mathrm{~L}, d_{M}\right\}$ respectively, where $N$ is the number of CPs, and $M$ is the number of CDs. Moreover, the contents, which the CDs in D want, constitute a library $\mathrm{F}=\left\{f_{1}, \mathrm{~L}, f_{k}, \mathrm{~L}, f_{K}\right\}$. If these CPs have these contents and they can establish D2D links with some CDs, the latter may well obtain the desired contents from some of these CPs based on $\mathrm{D} 2 \mathrm{D}$ communications. Once the request of a CD cannot be satisfied by any $\mathrm{CP}$, it will obtain 
its desired content from the BS. Note that, the BS can afford any required contents, and thus, it can be regarded as a CP. In this way, we define the set of the CPs who can establish the D2D links with $d_{i}$ as $\mathrm{P}\left(d_{i}\right)=\left\{p_{j} \mid R_{p_{j}, d_{i}} \geq R_{t h}\right\}$, where $R_{t h}$ is the minimal achievable rate of $d_{i}$, and $R_{p_{j}, d_{i}}$ is the achievable rate of the D2D link between $d_{i}$ and $p_{j}$. Here, we employ the underlying D2D communication sharing downlink resources with reuse mode. Then, we can leverage the existing resource allocation schemes, e.g., [3], to properly schedule the cellular links and coordinating their transmit power to mitigate the interference and derive the optimal link rate. Then, according to [24], $R_{p_{j}, d_{i}}$ can be expressed as

$$
R_{p_{j}, d_{i}}=\log _{2}\left(1+\frac{q_{p_{j}, d_{i}}^{c_{j i}} H_{p_{j}, d_{i}}^{c_{j i}}}{\sigma^{2}+q_{c_{j i}} H_{d_{i}}^{c_{j i}}}\right),
$$

where $q_{p_{j}, d_{i}}^{c_{j i}}$ is the transmit power of $p_{j}$ when it sends contents to $d_{i}$ via the D2D link which reuses the link of an ordinary cellular user $c_{j i}$, and $q_{c_{j i}}$ is the transmit power of BS for $c_{j i} . H_{p_{j}, d_{i}}^{c_{j i}}$ denotes the channel gain from $p_{j}$ to $d_{i}$ by reusing the downlink of $c_{j i}$, and $H_{d_{i}}^{c_{j i}}$ denotes the channel gain from the BS to $d_{i}$. They are modeled by the propagation channel model with path loss and frequency flat Rayleigh fading. $\sigma^{2}$ is the variance of the additive white Gaussian noise. Specifically, there is at most one D2D pair reusing the link of every ordinary cellular user.

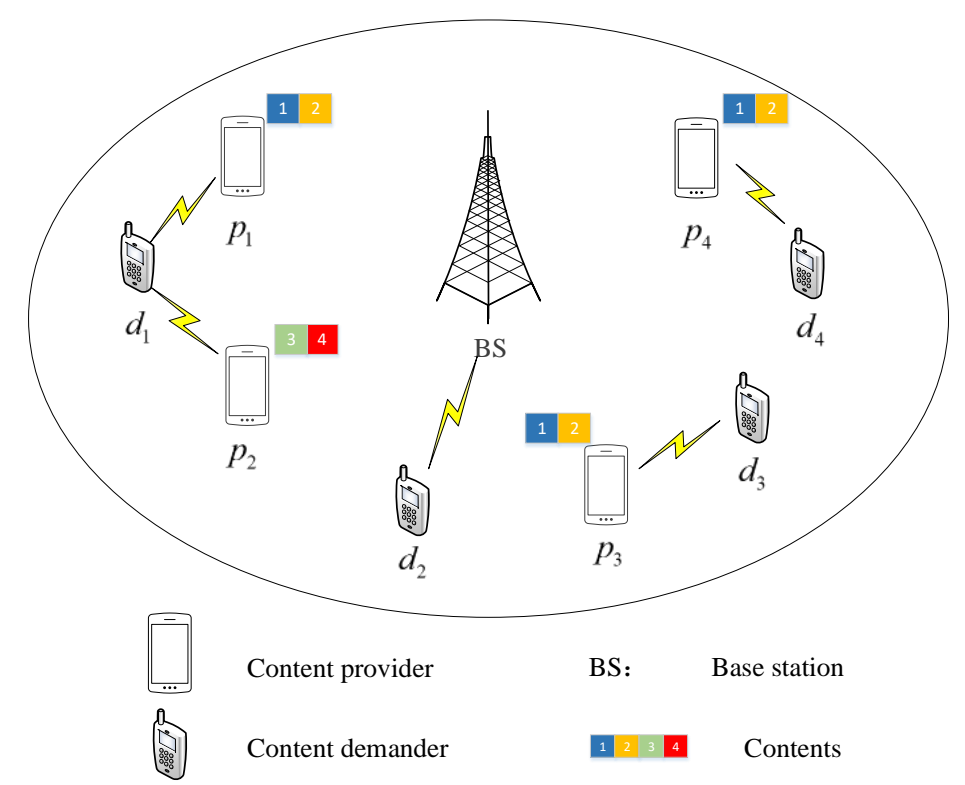

Fig. 1. The system model.

As mentioned above, the CPs need to make the most of their limited storage space by caching contents selectively to meet more content demands of CDs. Take Figure 1 as an example, $d_{1}, d_{2}$ and $d_{3}$ all requests $f_{1}, f_{2}, f_{3}$, and $f_{4}$ with a specified probability, and the probability is decreasing. $d_{3}$ can establish the D2D link with $p_{3}$, and thus $p_{3}$ selects to cache $f_{1}$ and $f_{2}$ to meet the content demand of $d_{3}$ as far as possible. Moreover, when the 
CDs can establish the D2D links with multiple CPs, these CPs need to cache diversified contents selectively by using the interaction among the CPs to avoiding the redundancy of caching contents and the waste of storage space. That is the collaborative caching. For example, $d_{1}$ can establish the D2D links with $p_{1}$ and $p_{2}$ respectively. Hence, $p_{1}$ and $p_{2}$ should cache these contents without repetition by exploiting the interaction between them. Thus, once $f_{1}$ and $f_{2}$ are cached in $p_{1}, p_{2}$ selects to cache $f_{3}$ or $f_{4}$ next based on the current caching situation of $p_{1}$. That is the collaborative caching, which can be exploited to make $p_{1}$ and $p_{2}$ cache different contents by the interaction between them to meet the content demands of $d_{1}$ and avoid the waste of storage space of $p_{1}$ and $p_{2}$.

\section{Social-Aware Collaborative Caching Model}

\subsection{Social Tie}

Since the CPs need to exploit their limited storage space to cache contents, and consume their energy to share contents, the CPs may not be willing to cache some contents requested by the others and share contents with them due to the selfish nature of CPs. To this end, how to stimulate the content caching and sharing is of importance. In fact, the CPs prefer to offer contents to some CDs who have stronger social relationships with them, such as friends and acquaintance. In this regard, the social information, which can be obtained from the social networks, can be adopted to stimulate the CPs to share contents.

According to [25], we employ the social tie $s_{d_{i}, p_{j}} \in[0,1]$ to characterize the strength of social relationship between $d_{i}$ and $p_{j}$. The larger the value of $s_{d_{i}, p_{j}}$, the stronger the social relationship between $d_{i}$ and $p_{j}$. Inspired by [25], we firstly can obtain the Jaccard coefficient $J_{d_{i}, p_{j}, y}$

$$
J_{d_{i}, p_{j}, y}=\frac{b_{d_{i}, y} \mathrm{I} b_{p_{j}, y}}{b_{d_{i}, y} \mathrm{U} b_{p_{j}, y}},
$$

where $b_{d_{i}, y}$ and $b_{p_{j}, y}$ stands for social factors of $d_{i}$ and $p_{j}$ respectively. Here, the social factor can be derived from the friend sets, the locations or the interests of $d_{i}$ and $p_{j}$. The social tie $s_{d_{i}, p_{j}}$ can be obtained by considering these social factors of $d_{i}$ and $p_{j}$. That is, it can be expressed as

$$
s_{d_{i}, p_{j}}=\sum_{y=0}^{W} w_{y} J_{d_{i}, p_{j}, y} .
$$

Here, $w_{y}$ denotes the relative weight of each social factor, and there are $W$ social factors influencing the social ties. In essence, the social tie in (3) indicates the similarity in some aspects. For example, when the social factor is derived from the interests of $d_{i}$ and $p_{j}$, the resulting social tie shows the interest similarity between them.

Similarly, the social tie $s_{d_{i}, d_{j}}$ between $d_{i}$ and $d_{j}$ can be described as

$$
S_{d_{i}, d_{j}}=\sum_{y=0}^{W} w_{y} J_{d_{i}, d_{j}, y},
$$


where $J_{d_{i}, d_{j}, y}=\frac{b_{d_{i}, y} \mathrm{I} b_{d_{j}, y}}{b_{d_{i}, y} \mathrm{U} b_{d_{j}, y}}$. Since the correlation of content demands of CDs is related t o the interest similarity between the CDs, the social ties can be used to characterize the content demands of individual CDs.

\subsection{User-Oriented Content Popularity}

To selectively cache contents, it is important for the CPs to know which contents the CDs want to request. In general, the demands of the CDs can be regarded as the content popularity, and obey the Zipf distribution, which represents the probability distribution of requests for the contents set $\mathrm{F}$ [17]. Thus, the probability $q_{f_{k}}$ that the content $f_{k}$ is requested is

$$
q_{f_{k}}=\frac{\left(1 / f_{k}\right)^{\lambda}}{\sum_{i=1}^{K}\left(1 / f_{i}\right)^{\lambda}}
$$

Here, $q_{f_{k}} \in[0,1]$ and $\sum_{k=1}^{K} q_{f_{k}}=1 . \lambda$ is the demand dominance factor [12]. The Zipf distribution is more skewed with larger $\lambda$, which means that a very large number of content demands focus on a very small number of contents.

Actually, the content demands of the CDs may be diverse. The aforementioned content popularity just characterizes the collective preferences from all the $\mathrm{CDs}$, rather than reflecting the diversity of content demands of individual CDs. Considering that the social ties between the CDs can be used to describe the correlation of content demands of CDs, by characterizing the interest similarity between the CDs, we try to define the user-oriented content popularity by combining the content popularity with the social ties. Importantly, the user-oriented content popularity $q_{d_{i}, f_{k}}$ is the probability that content $f_{k}$ is requested by $d_{i}$, so as to characterize various demands of the CDs.

Inspired by [17], we define the correlation value $h\left(U_{d_{i}}, V_{f_{k}}\right)$, which reflects the relatedness between $d_{i}$ and $f_{k}$. The larger the correlation value, the larger the probability that the content $f_{k}$ is requested by $d_{i}$. The correlation value $h\left(U_{d_{i}}, V_{f_{k}}\right)$ can be expressed as

$$
h\left(U_{d_{i}}, V_{f_{k}}\right)=\left(1-\left|U_{d_{i}}-V_{f_{k}}\right|\right)^{\left(1 / \beta^{3^{-1}}\right)} .
$$

Here, $h\left(U_{d_{i}}, V_{f_{k}}\right) \in[0,1]$. The BS assigns a variable $V_{f_{k}} \in[0,1]$ to $f_{k}$, and $d_{i}$ assigns a variable $U_{d_{i}} \in[0,1]$ to himself based on his interest. For example, if $d_{i}$ is interested in the basketball matches, and the content $f_{k}$ is related to the basketball matches, the correlation value $h\left(U_{d_{i}}, V_{f_{k}}\right)$ is large. Based on this, the user-oriented content popularity based on the correlation value can reflect the degree that individual CDs are interested in some contents. Different from that $\beta$ is a fixed parameter in [16], [17], we define $\beta=\mathbf{E}\left[s_{d_{i}, d_{j}}\right]$, which characterizes the average similarity of content demands of all CDs. The smaller the value of $\beta$, the worse the content demands of the CDs are correlated. 
Hence, the user-oriented content popularity $q_{d_{i}, f_{k}}$ can be represented as

$$
q_{d_{i}, f_{k}}=q_{f_{k}} \frac{h\left(U_{d_{i}}, V_{f_{k}}\right)}{\sum_{i=1}^{M} h\left(U_{d_{i}}, V_{f_{k}}\right)} .
$$

In this regard, the user-oriented content popularity $q_{d_{i}, f_{k}}$ can reflect different content demands of individual CDs to some extent, because it not only can reflect the popularity of contents for all the CDs based on the Zipf distribution, but also can characterize the diversity of content demands of individual CDs based on the interest of CDs.

\subsection{Collaborative Caching Model Based on User Preferences}

The collaborative caching is of importance to avoiding the redundancy of caching contents and the waste of storage space. With the goal of increasing the utilization ratio of the storage space, we propose the social-aware collaborative caching based on the user preferences. In general, the collaborative caching helps to cache more diversified contents by using the interaction among the CPs. The common way is to make use of the interaction among the caching situation of CPs. For example, in [8], the collaborative caching can be achieved by exploiting the content popularity and the current caching situation. However, the content popularity cannot describe the diversity of content demands of individual CDs. Recall that, the proposed user-oriented content popularity can better characterize various content demands of CDs. Hence, we try to add it to refine the collaborative caching model.

Specifically, we define the user preference of $d_{i}$ for content $f_{k}$ based on the user-oriented content popularity and the current caching situation as $G_{d_{i}, f_{k}}\left(x_{f_{k}, p_{j}}\right)$, which characterizes the caching value of the contents affected by various content demands of the CDs and the current caching situation of the CPs. It is expressed as

$$
G_{d_{i}, f_{k}}\left(x_{f_{k}, p_{j}}\right)=q_{d_{i}, f_{k}}\left[1-g_{d_{i}, f_{k}}\left(x_{f_{k}, p_{j}}\right)\right] .
$$

Here, $g_{d_{i}, f_{k}}\left(x_{f_{k}, p_{j}}\right)$ is the current caching situation of content $f_{k}$ within the range of D2D communications of $d_{i}$. It is expressed as

$$
g_{d_{i}, f_{k}}\left(x_{f_{k}, p_{j}}\right)=\frac{\sum_{p_{j} \in \mathrm{P}\left(d_{i}\right)} x_{f_{k}, p_{j}}}{\sum_{p_{j} \in \mathrm{P}\left(d_{i}\right)} c_{p_{j}}^{d_{i}}} .
$$

Here, $x_{f_{k}, p_{j}} \in\{0,1\}$ is the caching indicator. If $x_{f_{k}, p_{j}}=1$, the content $f_{k}$ is cached by $p_{j}$. Otherwise the content $f_{k}$ is not cached by $p_{j}$. Besides, $c_{p_{j}}^{d_{i}} \in\{0,1\}$ is the indicator of positional relation between $d_{i}$ and $p_{j}$, which is determined by the BS. If $p_{j}$ is within the range of D2D communications of $d_{i}, c_{p_{j}}^{d_{i}}=1$. Otherwise $c_{p_{j}}^{d_{i}}=0$. Specifically, before the content is actually requested and answered through D2D communications, the BS collects and updates the location information of the CPs and the CDs, and determines the transmit power of CPs participating in D2D content sharing and the minimal achievable rate $R_{t h}$ of CDs. Then, the achievable rate $R_{p_{j}, d_{i}}$ of the D2D link between $d_{i}$ and $p_{j}$ can be obtained. 
Finally, the BS determines the value of $c_{p_{j}}^{d_{i}}$ based on the following rules: If $R_{p_{j}, d_{i}} \geq R_{t h}$, $p_{j}$ is within the range of $\mathrm{D} 2 \mathrm{D}$ communications of $d_{i}$, and $c_{p_{j}}^{d_{i}}=1$. Otherwise $p_{j}$ is without the range of D2D communications of $d_{i}$, and $c_{p_{j}}^{d_{i}}=0$.

In this regard, we revise the collaborative caching model in [8] by adding the user preference. Adding the user preference facilitates the interaction among the CPs, so that CPs can cache more diversified contents and meet more content demands of CDs. As such, the advantage of our proposed collaborative caching model is to provide high cache gain and avoid that some popular contents are cached too many times.

\subsection{Problem Formulation}

Guided by the proposed collaborative caching model, we firstly define the social-aware rate as a metric, which characterizes the achievable contents download rate by the consideration of the impacts of the social ties, the caching indicators and the user preferences, as shown in Fig. 2. Then, when $p_{j}$ can establish the D2D link with $d_{i}$, the social-aware rate of content $f_{k}$ cached by $p_{j}$ for $d_{i}$ can be expressed as

$$
\operatorname{SR}\left(x_{f_{k}, p_{j}}\right)=s_{d_{i}, p_{j}} R_{p_{j}, d_{i}} x_{f_{k}, p_{j}} G_{d_{i}, f_{k}}\left(x_{f_{k}, p_{j}}\right) .
$$

Hence, the social-aware collaborative caching problem based on the social-aware rate can be formulated as

$$
\begin{aligned}
& \max _{x_{f_{k}, p_{j}}} \sum_{i=1}^{M} \sum_{j=1}^{N} \sum_{k=1}^{K} S R\left(x_{f_{k}, p_{j}}\right) \\
& \text { s.t. } \sum_{k=1}^{K} x_{f_{k}, p_{j}} \leq C_{p_{j}}, j=1,2, \mathrm{~L}, N \\
& x_{f_{k}, p_{j}} \in\{0,1\}, \forall 1 \leq k \leq K, 1 \leq j \leq N
\end{aligned}
$$

Here, $\sum_{k=1}^{K} x_{f_{k}, p_{j}} \leq C_{p_{j}}$ is the constraint of limited storage space of $p_{j}$, where $C_{p_{j}}$ is the maximal storage space of $p_{j}$.

Note that, the above social-aware collaborative caching problem based on the user preferences is an integer programming problem to maximize the sum of social-aware rate of all contents for all the CPs and all the CDs, subject to some constraints, e.g., the constraint of the limited storage space. As shown in [12], it is typically NP-hard, which usually leads to computational intractability. In practice, it is more available to search some algorithms to strike a balance between the optimal solution and the implementation complexity [24]. 


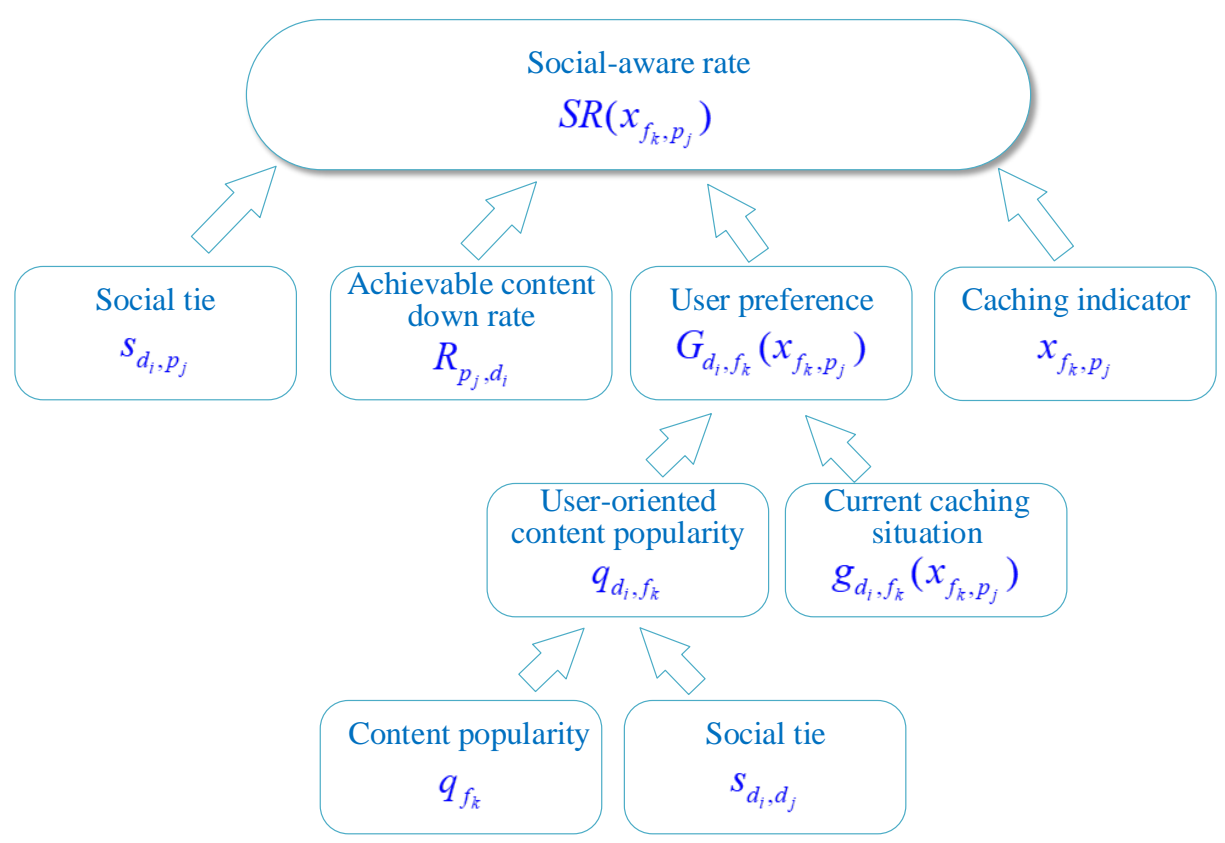

Fig. 2. The problem formulation.

\section{Monotone Submodular Function over One Matroid Constraint}

In this section, we show that the social-aware collaborative caching problem in (11) can be computationally efficiently simplified to the maximization problem of a monotone submodular function over a matroid constraint. In fact, for the submodular maximization problem over matroid constraints, there exist a series of effective approximation algorithms with provable approximation ratios, which has been found a wide range of applications in wireless networks, especially D2D networks. Hence, the simplification can help to search some algorithms to solve the proposed social-aware collaborative caching problem in (11).

To prove that the social-aware collaborative caching problem based on the user preferences in (11) is the maximization problem of a monotone submodular function over a matroid constraint, we define a ground set:

$$
\begin{aligned}
\mathrm{S} & =\left\{s_{1}^{1}, \mathrm{~L}, s_{k}^{1}, \mathrm{~L}, s_{K}^{1}, \mathrm{~L}, s_{1}^{N}, \mathrm{~L}, s_{k}^{N}, \mathrm{~L}, s_{K}^{N}\right\} \\
& =\left\{s_{1}, \mathrm{~L}, s_{j}, \mathrm{~L}, s_{N}\right\}
\end{aligned}
$$

Here, $s_{k}^{j}$ denotes the indicator that content $f_{k}$ is cached in $p_{j}$, and $s_{j}=\left\{s_{1}^{j}, \mathrm{~L} s_{k}^{j}, \mathrm{~L}, s_{K}^{j}\right\}$ denotes the set of the indicators of all the contents cached in $p_{j}$.

According to the definitions of matroid and submodular function (refer to [12]), we have the following theorems.

Theorem 1. The constraint of the limited storage space of CPs $\sum_{k=1}^{K} x_{f_{k}, P_{j}} \leq C_{p_{j}}$ can be formulated as a matroid on the ground set $\mathrm{S}$ defined in (12).

Proof. In the constraint of the limited storage space of CPs $\sum_{k=1}^{K} x_{f_{k}, P_{j}} \leq C_{p_{j}}$, the social-aware collaborative caching set is expressed by $X \subseteq \mathrm{S}$. We can notice that $s_{k}^{j} \in X$ if and only if $x_{f_{k}, P_{j}}=1$. Hence the constraint can be expressed as 


$$
\mathrm{T}=\left\{X \subseteq \mathrm{S}:\left|X \mathrm{I} \quad s_{j}\right| \leq C_{p_{j}}, j=1,2, \mathrm{~L}, N\right\}
$$

Compared with the definition of matroid in [12], we can notice that the constraint of the limited storage space of $\mathrm{CPs}$ forms a matroid $\mathrm{M}=(\mathrm{S}, \mathrm{T})$.

Theorem 2. The object function $\sum_{i=1}^{M} \sum_{j=1}^{N} \sum_{k=1}^{K} S R\left(x_{f_{k}, p_{j}}\right)$ is a monotone submodular function.

Proof. We formulate the object function as $f(X)=\sum_{i=1}^{M} \sum_{j=1}^{N} \sum_{k=1}^{K} S R\left(x_{f_{k}, p_{j}}\right)$, where $\operatorname{SR}\left(x_{f_{k}, p_{j}}\right)$ is related to the current caching situation.

Firstly, we prove the monotonicity. Let us consider two placement sets $A$ and $A \cup\{e\}$, where $e \in \mathrm{S} \backslash A$. As a result, we have

$$
\begin{gathered}
f(A)=\sum_{i=1}^{M} \sum_{j=1}^{N} \sum_{k=1}^{K} S R\left(x_{f_{k}, P_{j}}\right) \\
f(A \cup\{e\})=\sum_{i=1}^{M} \sum_{j=1}^{N} \sum_{k=1}^{K} S R\left(x_{f_{k}, p_{j}}\right)+\sum_{i=1}^{M} s_{d_{i}, p_{j}} R_{p_{j}^{\prime}, d_{i}} x_{f_{k}^{\prime}, p_{j}^{\prime}} G_{d_{i}, f_{k}^{\prime}}^{\prime}\left(x_{f_{k}, p_{j}}\right) \\
=\sum_{i=1}^{M} \sum_{j=1}^{N} \sum_{k=1}^{K} S R\left(x_{f_{k}, p_{j}}\right)+\sum_{i=1}^{M} s_{d_{i}, p_{j}} R_{p_{j}^{\prime}, d_{i}} x_{f_{k}^{\prime}, p_{j}^{\prime}} q_{d_{i}, f_{k}^{\prime}}\left[1-g_{d_{i}, f_{k}^{\prime}}^{\prime}\left(x_{f_{k}, p_{j}}\right)\right]
\end{gathered}
$$

Here, $G_{d_{i}, f_{k}^{\prime}}^{\prime}\left(x_{f_{k}, p_{j}}\right)$ and $g_{d_{i}, f_{k}^{\prime}}^{\prime}\left(x_{f_{k}, p_{j}}\right)$ are related to the current caching situation. In other words, they are related to the caching situation of the placement set $A$. Because $s_{d_{i}, p_{j}^{j}} \geq 0$, $R_{p_{j}^{\prime}, d_{i}} \geq 0, \quad x_{f_{k}^{\prime}, p_{j}^{\prime}} \geq 0, \quad q_{d_{i}, f_{k}^{\prime}} \geq 0$, and $0 \leq g_{d_{i}^{\prime}, f_{k}^{\prime}}^{\prime}\left(x_{f_{k}, p_{j}}\right) \leq 1$, we can conclude that $0 \leq 1-g_{d_{i}, f_{k}^{\prime}}^{\prime}\left(x_{f_{k}, p_{j}}\right) \leq 1$ and $f(A \cup\{e\}) \geq f(A)$. Namely, the object function is a monotone function.

Secondly, we prove that the object function is a submodular function. We consider three placement sets $A, B$ and $e$, where $A \subseteq B$ and $e \in \mathrm{S} \backslash B$. Based on (14), (15), we have

$$
\begin{aligned}
& f(A \cup\{e\})-f(A)=\sum_{i=1}^{M} s_{d_{i}, p_{j}} R_{p_{j}, d_{i}} x_{f_{k}^{\prime}, p_{j}} q_{d_{i}, f_{k}^{\prime}}\left[1-g_{d_{i}, f_{k}^{\prime}}^{\prime}\left(x_{f_{k}, p_{j}}\right)\right] \\
& f(B \cup\{e\})-f(B)=\sum_{i=1}^{M} s_{d_{i}, p_{j}} R_{p_{j}, d_{i}} x_{f_{k}^{\prime}, p_{j}} q_{d_{i}, f_{k}^{\prime}}\left[1-g_{d_{i}, f_{k}^{\prime}}^{\prime \prime}\left(x_{f_{k}, p_{j}}\right)\right]
\end{aligned}
$$

Here, $g_{d_{i}, f_{k}^{\prime}}^{\prime}\left(x_{f_{k}, p_{j}}\right)$ is the caching situation of content $f_{k}$ based on the placement set $A$, and $g_{d_{i}, f_{k}^{\prime}}^{\prime \prime}\left(x_{f_{k}, p_{j}}\right)$ is the caching situation of content $f_{k}$ based on the placement set $B$. Due to $A \subseteq B$, it is obvious that $g_{d_{i}, f_{k}^{\prime}}^{\prime}\left(x_{f_{k}, p_{j}}\right) \leq g_{d_{i}, f_{k}^{\prime}}^{\prime \prime}\left(x_{f_{k}, p_{j}}\right)$. As a result, we can have

$$
f(A \cup\{e\})-f(A) \geq f(B \cup\{e\})-f(B)
$$

Compared with the definition of submodular function in [12], the object function is a submodular function.

Hence, we can conclude that the proposed social-aware collaborative caching problem in (11) is the maximization problem of a monotone submodular function subject to a matroid constraint. Based on the simplification, we can design some algorithms with the different provable approximation gaps and computational complexity. 


\section{The Social-Aware Collaborative Caching Algorithms Based on Greedy Algorithms}

In this section, we firstly employ the aforementioned simplification to design a low-complexity algorithm with a constant-factor approximation gap based the standard greedy algorithm. Secondly, we design an algorithm with higher approximation ratio based the continuous greedy algorithm due to the relationship between the maximization problem of a monotone submodular function over a matroid constraint and the maximization problem of a monotone smooth submodular function over a matroid polytope constraint.

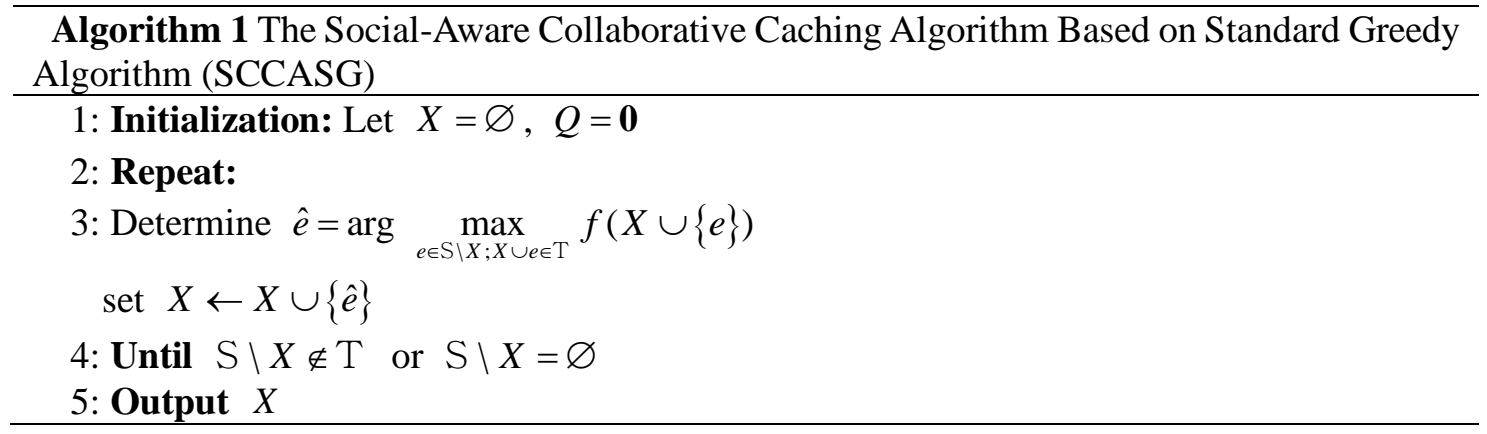

By leveraging the properties of submodularity and matroid constraint, we design the social-aware collaborative caching algorithm based on standard greedy algorithm, called as SCCASG for simplify, and the detailed description is presented in Algorithm 1. It starts with an empty set, and adds the new element which can obtain the highest marginal value to the set at each step. Generally, when the highest marginal value is zero, the greedy algorithm terminates. That is because the marginal value of submodular function of adding a new element to the set will not increase as the set becomes larger. In Algorithm 1, once all the users are full to the storage space, the algorithm stops because of the constraint of limited storage space.

According to [26], for the maximization problem of a monotone submodular function subject to a matroid constraint, Algorithm 1 achieves a suboptimal solution with a factor $1 / 2$ approximation guarantee. In addition, the final social-aware collaborative caching scheme can be obtained through at most $N K$ iterations. In the first iteration, we need compare the value of $N K$ situations, and the number of situations decrease progressively as the number of iterations increases. Hence, the complexity of Algorithm 1 is $O\left(N^{2} K^{2}+N K\right)$.

Algorithm 2 The Social-Aware Collaborative Caching Algorithm Based on Continuous Greedy Algorithm (SCCACG)

1: Initialization: $t=0$, Let $\phi=\frac{1}{9 K^{2}}, y(0)=\mathbf{0}$

2: while $t<1$ do

3: Calculate $\Delta z_{f_{k}, p_{j}}(t)$, which is the estimate of $\mathbf{E}\left[f\left(\left\{s_{k}^{j}\right\} \mathrm{UY}(t)\right)-f(\mathrm{Y}(t))\right]$, obtained by taking the average of $\frac{10}{\phi^{2}}[1+\ln (N K)]$ samples of $\mathrm{Y}(t)$, where $\mathrm{Y}(t)$ contains each $s_{k}^{j}$ independently with probability $y_{f_{k}, p_{j}}(t)$;

4: Determine $\left(f_{k}, p_{j}\right)=\arg \max _{f_{k} \in \mathrm{F}, p_{j} \in \mathrm{P}: \mathbb{X}^{(t-1)} \in \mathrm{T}} \Delta z_{f_{k}, p_{j}}(t)$ 


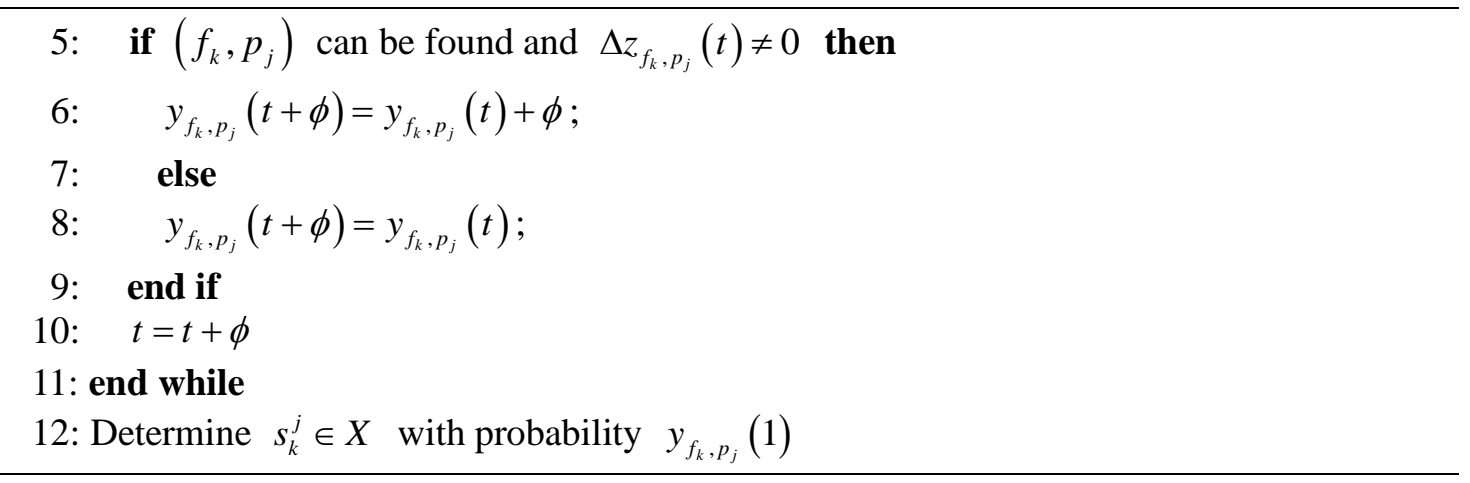

Moreover, the continuous greedy algorithm can be also used to solve the proposed problem with higher approximation ratio. According to [27], for the problem $\max \{f(X): X \in \mathrm{T}\}$, where $f: 2^{\mathrm{S}} \rightarrow \mathrm{R}$ is a monotone submodular function given by a value oracle, and $\mathrm{M}=(\mathrm{S}, \mathrm{T})$ is a matroid given by a membership oracle, a common way of replacing a discrete function by a continuous function is the multilinear extension $F(y)=\mathbf{E}[f(X)]$, where the new continuous problem is describe as $\max \{F(y): y \in P(\mathrm{M})\}$, and $F:[0,1]^{\mathrm{S}} \rightarrow \mathrm{R}$ is a monotone smooth submodular function. However, it is hard to evaluate $F(y)$ accurately. We can only approximate the value of $F(y)$ by random sampling. Hence, the continuous greedy process and the pipage rounding technique can be exploited to solve the original problem with a provable approximation ratio. That is, we exploit the relationship between the problem $\max \{f(X): X \in \mathrm{T}\}$ and $\max \{F(y): y \in P(\mathrm{M})\}$ to design another algorithm with higher approximation ratio based the continuous greedy algorithm, called as SCCACG for simplify, and the sketch is shown in Algorithm 2. In this algorithm, we firstly adopt a continuous greedy process to approximate $\max \{F(y): y \in P(\mathrm{M})\}$. Secondly, we leverage the pipage rounding technique to convert a fractional solution to a discrete solution.

According to [12] and [27], for the maximization problem of a monotone submodular function subject to a matroid constraint, Algorithm 2 achieves a suboptimal solution with a $\left(1-1 / e^{)}\right.$-approximation, and its complexity is $O\left((N K)^{8}\right)$. In particular, the $(1-1 / e)$-approximation is optimal in polynomial time [27].

In summary, Algorithm 1 has lower complexity although its approximation ratio is lower, and Algorithm 2 can achieve the optimal solution in polynomial time although its complexity is higher.

\section{Numerical Results}

In this section, we provide the simulation results to verify the performance of the SCCASG scheme and the SCCACG scheme we proposed. In the simulation, we establish the channel model, i.e., the channel gain $H_{j, i}=\left(d_{j, i}\right)^{-\alpha}\left|h_{j, i}\right|^{2}$, where $d_{j, i}$ is the distance between the CD and the CP, $\alpha=3$ is the path loss exponent, and $h_{j, i}$ is the unitary power, Rayleigh fading 
channel coefficient [24]. Besides, for comparison, we give some caching schemes:

1) Scheme 1: there doesn't exist collaborative caching among the CPs, and each content provider selects the most popular contents based on Zipf distribution to cache [28]. Scheme 1 is introduced in order to reflect the advantages of collaborative caching.

2) Scheme 2: the collaborative caching is only achieved by exploiting the content popularity and the current caching proportion of different contents [8]. Scheme 2 is exploited for showing the difference between our collaborative caching schemes and other collaborative caching scheme.

3) Scheme 3: this scheme is similar to our schemes, but the user-oriented content popularity is replaced by the Zipf distribution. Scheme 3 is used to verify the advantages of user-oriented content popularity in our schemes.

4) Scheme 4: this scheme is similar to our schemes, but the social ties are not introduced into this scheme. Scheme 4 is used to prove the effect of social information.

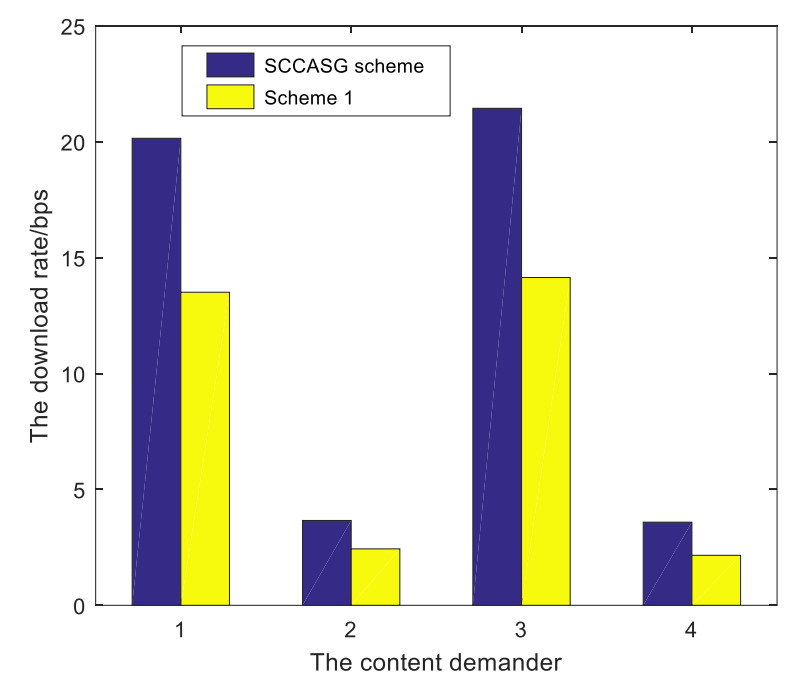

Fig. 3. The comparison of download rate of all contents for each CD between SCCASG and Scheme 1.

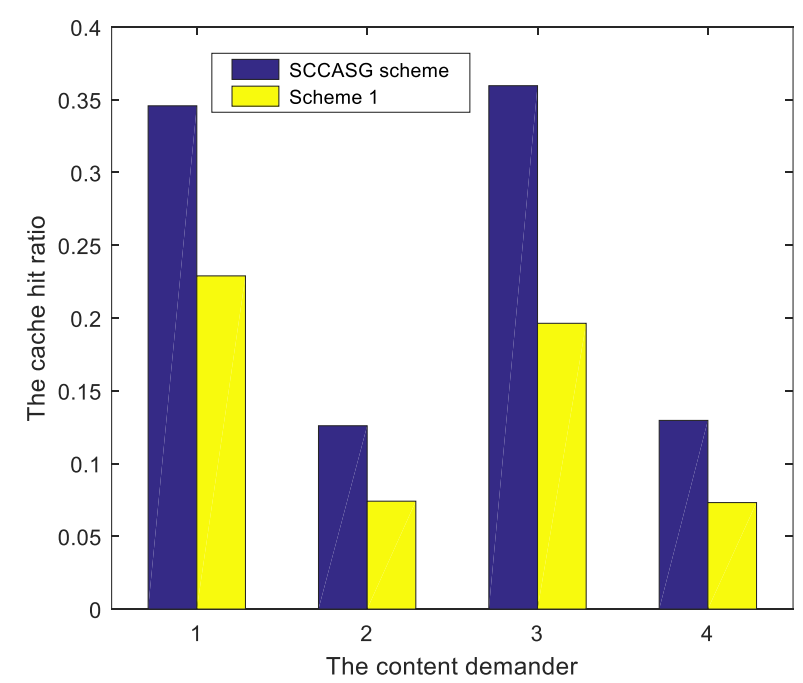

Fig. 4. The comparison of cache hit ratio for each CD between SCCASG and Scheme 1. 


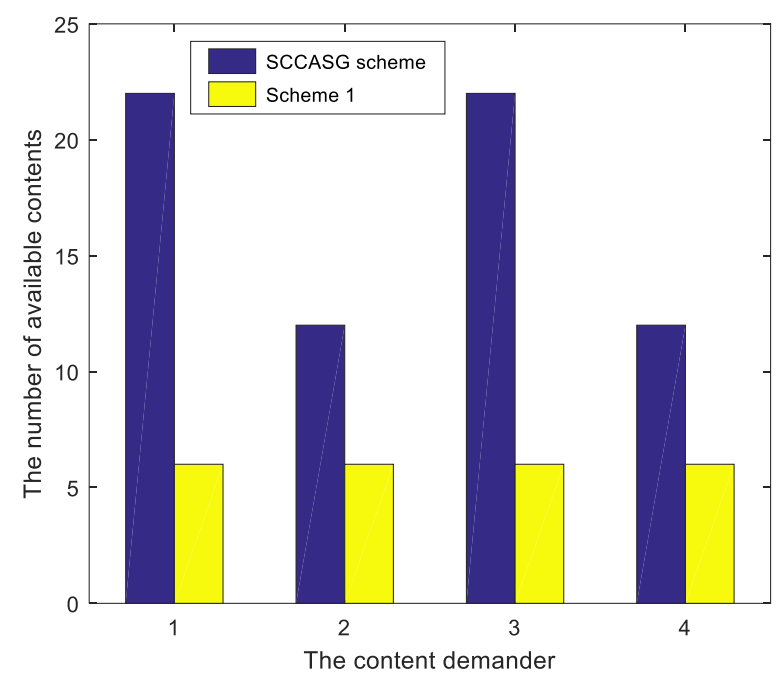

Fig. 5. The comparison of number of available contents for each CD between SCCASG and Scheme 1.

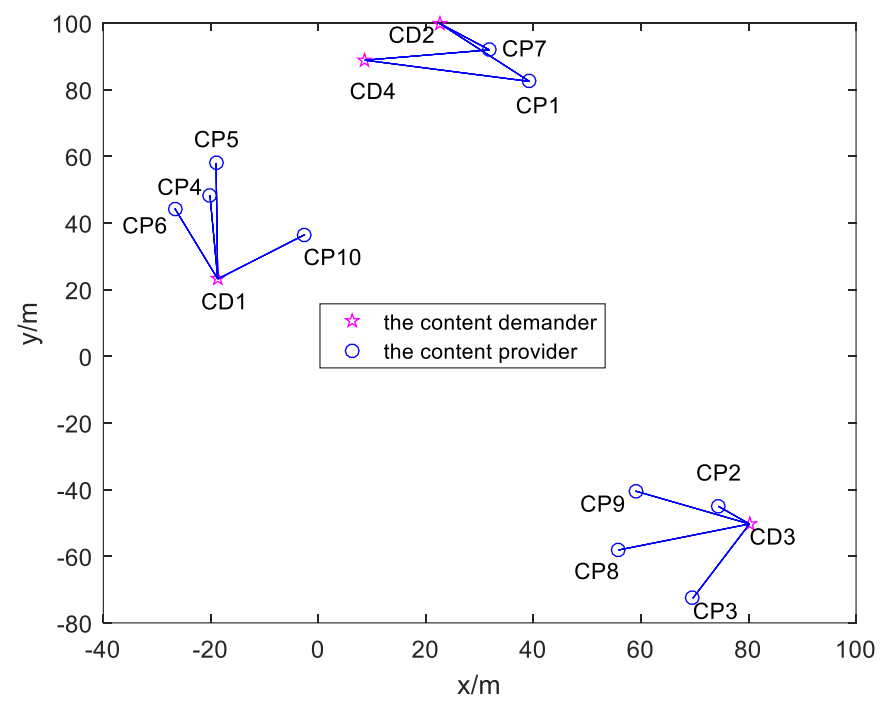

Fig. 6. The location relationship between the CPs and the CDs.

In Fig. 3, Fig. 4 and Fig. 5, we show the comparison of the performance between the SCCASG scheme and Scheme 1, when $M=4, N=10, K=40$. As shown in Fig. 3 and Fig. 4, it is obvious that the download rate and the cache hit ratio for the CDs in the SCCASG scheme are superior to these in Scheme 1. In addition, Fig. 5 reveals that these CDs in the SCCASG scheme can obtain not only larger download rate and cache hit ratio, but also more kinds of contents. That is because the user-oriented content popularity is exploited to characterize properly the diversity of content demands of individual CDs and improve the availability of preset contents in the SCCASG scheme. Besides, the SCCASG scheme makes the CPs cache more different contents by exploiting the social-aware collaborative caching. As for $C D_{2}$ and $\mathrm{CD}_{4}$, their download rate and cache hit ratio in the two schemes are low. Because there is only two CPs within the range of D2D communications of $C D_{2}$ or $C D_{4}$, as shown in Fig. 6. In Fig. 6, we show the location relationships between the CPs and the CDs in the SCCASG 
scheme shown in Fig. 3, Fig. 4 and Fig. 5. The blue lines indicate the distance between the $\mathrm{CPs}$ and the CDs is within the range of D2D communications.

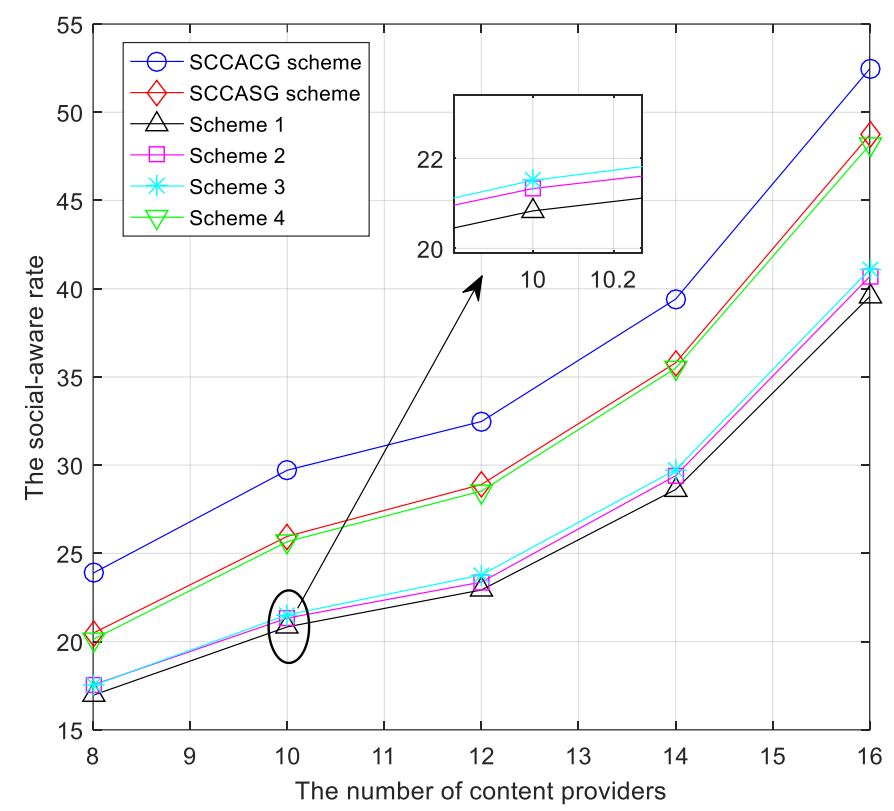

Fig. 7. The comparison of social-aware rate CPs can provide with different number of CPs.

Fig. 7 compares the social-aware rate CPs can provide in our schemes with that in Scheme 1, Scheme 2, Scheme 3 and Scheme 4 versus the number of CPs $N$, when $M=4, K=40$. Clearly, for these schemes, higher social-aware rate can be obtained with larger number of CPs, when other system parameters are fixed. Because more CPs can satisfy more content demands with more storage space. Moreover, our schemes are greater than the other four schemes with different number of CPs. Specifically, in Scheme 1, the CPs can only cache those most popular contents, and cannot achieve the collaborative caching, which will lead to that some contents are cached too many times and the performance is lower. In Scheme 2, the collaborative caching is only achieved by exploiting the content popularity and the current caching proportion of different contents, and it ignores the influence of social information and diversity of content demands of CDs, which will lead to lower social-aware rate. In Scheme 3, the user-oriented content popularity is replaced by the Zipf distribution so that it is hard to meet different content demands of CDs, which will lead to worse performance. In Scheme 4, the selfish nature of users is ignored, which will result that the social-aware rate the CPs can provide decreases. Besides, the performance of Scheme 3 and Scheme 4 is superior to that of Scheme 1 and Scheme 2, which verities the advantages of our collaborative caching method. In particular, the SCCACG scheme is greater than the SCCASG scheme, and the SCCACG scheme is an optimal solution in polynomial time. 


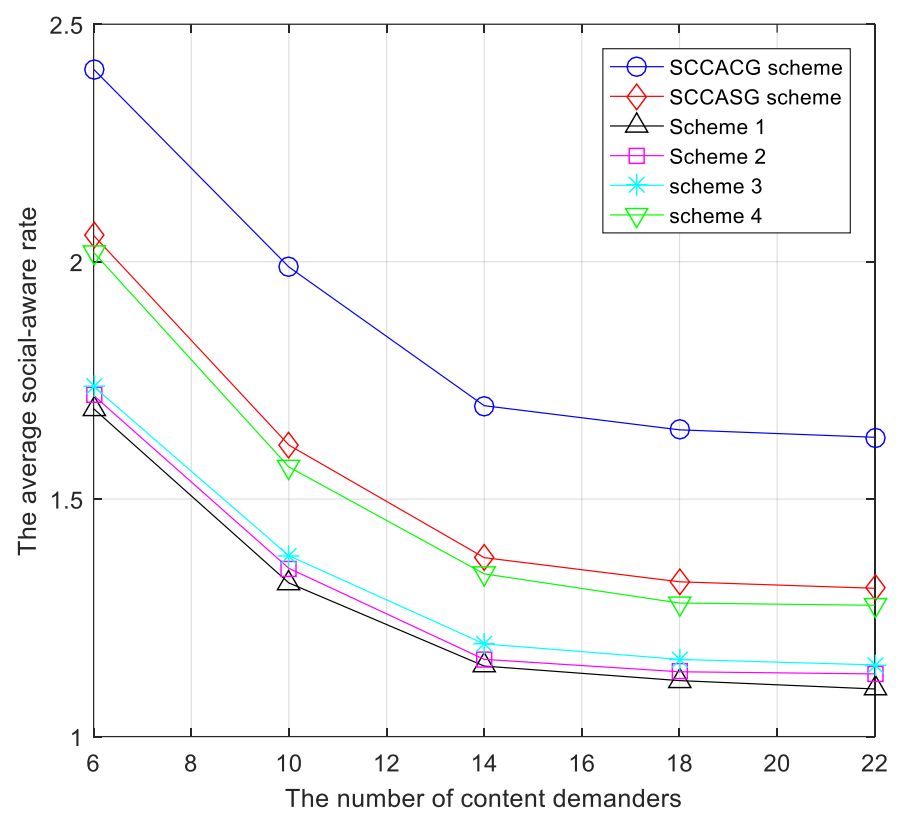

Fig. 8. The comparison of average social-aware rate CPs can provide with different number of CDs.

In Fig. 8 and Fig. 9, we illustrate the effect of the number of CDs and the demand dominance factor, when $N=10, K=40$. The performance is expressed as the average social-aware rate CPs can provide. The average social-aware rate is larger, which means that the scheme can employ less CPs to provide the CDs larger social-aware rate. As shown in these two figures, the performance of our schemes is greater than that of other four schemes with different number of CDs and demand dominance factor, of which the performance of the SCCACG scheme is greater than that of the SCCASG scheme. In addition, Fig. 8 shows that for these schemes, greater performance can be obtained with smaller number of CDs, when other system parameters are fixed. Because more content demands may not be satisfied with more CDs. Fig. 9 reveals that the schemes with larger demand dominance factor can obtain higher performance, when other system parameters are fixed. Because the content popularity distribution is more skewed with larger demand dominance factor, which means that the most content demands focus on these quite popular contents, and then these quite popular contents are selected to be cached in CPs in these schemes, which can satisfy more content demands of CDs. In other word, the limited storage space of CPs can satisfy more content demands when the content demands distribution is more skewed. In summary, the performance of our schemes is higher than that of other four schemes, with different system parameters. 


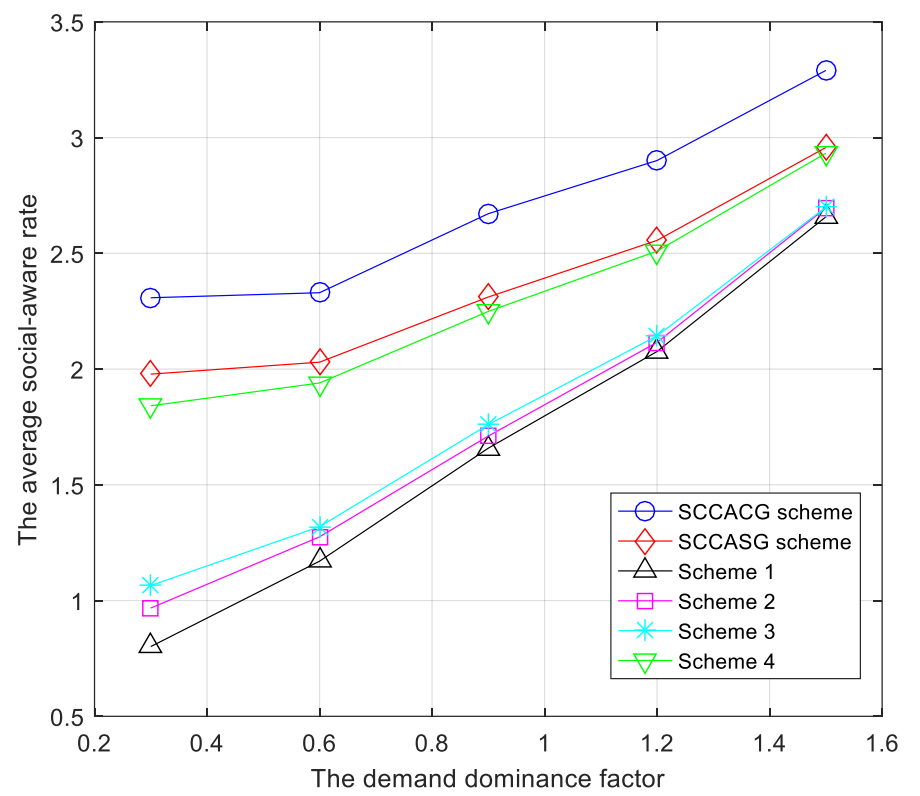

Fig. 9. The comparison of average social-aware rate CPs can provide with different $\lambda$.

\section{Conclusion}

Inspired by D2D content sharing, we focuse on the social-aware collaborative caching based on user preferences in $\mathrm{D} 2 \mathrm{D}$ content sharing scenarios. We propose the social-aware rate, which characterizes the achievable contents download rate affected by the social ties, the caching indicators, and the user preferences. In particular, the user preferences are defined to characterize the caching values of the contents for different CDs, which is composed of the user-oriented content popularity and the current caching situation. Then, with the goal of maximizing the sum of social-aware rate, we model the social-aware collaborative caching problem based on the user preferences to make a trade-off between the redundancy of caching contents and the cache hit ratio. Due to its intractability, it is simplified to the maximization of a monotone submodular function over a matroid constraint. Then, the social-aware collaborative caching algorithms are designed by leveraging the standard and continuous greedy algorithms respectively. We provide the simulation results to illustrate the performance of the schemes we proposed. In our ongoing work, we will pursue different collaborative caching schemes with higher performance.

\section{References}

[1] P. Lin, Q. Song, Y. Yu, and A. Jamalipour, "Extensive cooperative caching in D2D integrated cellular networks," IEEE Communications Letters, vol. 21, no. 9, pp. 2101-2104, September, 2017. Article (CrossRef Link)

[2] Y. Li, M. C. Gursoy, and S. Velipasalar, "A delay-aware caching algorithm for wireless D2D caching networks," in Proc. of IEEE INFOCOM WKSHPS, pp. 456-461, May 1-4, 2017.

Article (CrossRef Link) 
[3] D. Wu, Y. Cai, R. Q. Hu, Y. Qian, "Dynamic distributed resource sharing for mobile D2D communications," IEEE Transactions on Wireless Communications, vol. 14, no. 10, pp. 5417-5429, October, 2015. Article (CrossRef Link)

[4] D. Wu, J. Wang, R. Q. Hu, Y. Cai, "Energy-efficient resource sharing for mobile device-to-device multimedia communications," IEEE Transactions on Vehicular Technology, vol. 63, no. 5, pp. 2093-2103, June, 2014. Article (CrossRef Link)

[5] D. Wu, L. Zhou, Y. Cai, and Y. Qian, "Collaborative caching and matching for D2D content sharing," IEEE Wireless Communications, vol. 25, no. 3, pp. 43-49, June, 2018. Article (CrossRef Link)

[6] D. Wu, L. Zhou, Y. Cai, H. Chao, and Y. Qian, "Physical-social-aware D2D content sharing networks: a provider-demander matching Game," IEEE Transactions on Vehicular Technology, vol. 67, no. 8, pp. 7538-7549, August, 2018. Article (CrossRef Link)

[7] M. Ji, G. Caire, and A. F. Molisch, "Wireless device-to-device caching networks: basic principles and system performance," IEEE Journal on Selected Areas in Communications, vol. 34, no. 1, pp. 176-189, January, 2016. Article (CrossRef Link)

[8] J. Jiang, S. Zhang, B. Li, and B. Li, "Maximized cellular traffic offloading via device-to-device content sharing," IEEE Journal on Selected Areas in Communications, vol. 34, no. 1, pp. 82-91, January, 2016. Article (CrossRef Link)

[9] N. Golrezaei, P. Mansourifard, A. Molisch, and A. Dimakis, "Base-station assisted device-to-device communications for high-throughput wireless video networks," IEEE Transactions on Wireless Communications, vol. 13, no. 7, pp. 3665-3676, July, 2014. Article (CrossRef Link)

[10] D. Malak, M. Al-Shalash, J. G. Andrews, "Spatially correlated content caching for device-to-device communications," IEEE Transactions on Wireless Communications, vol. 17, no. 1, pp. 56-70, January, 2018. Article (CrossRef Link)

[11] M. Gregori, J. Gomez-vilardebo, J. Matamoros, and D. Gunduz, "Wireless content caching for small cell and D2D networks," IEEE Journal on Selected Areas in Communications, vol. 34, no. 5, pp. 1222-1234, May, 2016. Article (CrossRef Link)

[12] K. Shanmugam, N. Golrezaei, A. G. Dimakis, A. F. Molisch, and G. Caire, "FemtoCaching: wireless content delivery through distributed caching helpers," IEEE Transactions on Information Theory, vol. 59, no. 12, pp. 8402-8413, December, 2013. Article (CrossRef Link)

[13] N. Golrezaei, A. F. Molisch, A. G. Dimakis, and G. Caire, "Femtocaching and device-to-device collaboration: a new architecture for wireless video distribution," IEEE Communications Magazine, vol. 51, no. 4, pp. 142-149, April, 2013. Article (CrossRef Link)

[14] A. Tatar, M. D. de Amorim, S. Fdida, and P. Antoniadis, "A survey on predicting the popularity of web content," Journal of Internet Services and Applications, vol. 5, no. 1, pp. 1-20, December, 2014. Article (CrossRef Link)

[15] Y. Guo, L. Duan, and R. Zhang, "Cooperative local caching and file sharing under heterogeneous file preferences," in Proc. of 2016 IEEE International Conference on Communications, pp. 1-6, May 22-27, 2016. Article (CrossRef Link)

[16] M. Leconte, G. Paschos, L. Gkatzikis, M. Draief, S. Vassilaras, and S. Chouvardas, "Placing dynamic content in caches with small population," in Proc. of IEEE INFOCOM 2016-The 35th Annual IEEE International Conference on Computer Communications, pp. 1-9, April 10-14, 2016. Article (CrossRef Link)

[17] B. Chen, C. Yang, "Caching policy optimization for D2D communications by learning user preference," in Prof. of 2017 IEEE 85th Vehicular Technology Conference, pp. 1-6, June 4-7, 2017. Article (CrossRef Link)

[18] X. Li, T. Jiang, S. Cui, J. An, and Q. Zhang, "Cooperative communications based on rateless network coding in distributed MIMO systems," IEEE Wireless Communications, vol. 17, no. 3, pp. 60-67, June, 2010. Article (CrossRef Link)

[19] B. Chen, C. Yang, and G. Wang, "Cooperative device-to-device communications with caching," in Proc. of 2016 IEEE 83rd Vehicular Technology Conference, pp. 1-5, May 15-18, 2016. Article (CrossRef Link) 
[20] Z. Yang, H. Tian, S. Fan, and G. Chen, "Dynamic incentive design in content dissemination process through D2D communication," IEEE Communications Letters, vol. 21, no. 8, pp. 1799-1802, August, 2017. Article (CrossRef Link)

[21] E. Bastug, M. Bennis, and M. Debbah, "Living on the edge: The role of proactive caching in $5 \mathrm{~g}$ wireless networks," IEEE Communications Magazine, vol. 52, no. 8, pp. 82-89, August, 2014. Article (CrossRef Link)

[22] B. Bai, L. Wang, Z. Han, W. Chen, and T. Svensson, "Caching based socially-aware D2D communications in wireless content delivery networks: a hypergraph framework," IEEE Wireless Communications, vol. 23, no. 4, pp. 74-81, August, 2016. Article (CrossRef Link)

[23] C. Ma, M. Ding, and H. Chen, "Socially aware caching strategy in device to device communication networks," IEEE Transactions on Vehicular Technology, vol. 67, no. 5, pp. 4615-4629, May, 2018. Article (CrossRef Link)

[24] D. Wu, L. Zhou, and Y. Cai, "Social-aware rate based content sharing mode selection for D2D content sharing scenarios," IEEE Transactions on Multimedia, vol. 19, no. 11, pp. 2571-2582, November, 2017. Article (CrossRef Link)

[25] K. Zhu, W. Zhi, L. Zhang, X. Chen, and X. Fu, "Social-aware incentivized caching for D2D communications," IEEE Access, vol. 4, pp. 7585-7593, October, 2016. Article (CrossRef Link)

[26] M. Liu, O. Tuzel, S. Ramalingam, and R. Chellappa, "Entropy-rate clustering: cluster analysis via maximizing a submodular function subject to a matroid constraint," IEEE Transactions on Pattern Analysis and Machine Intelligence, vol. 36, no. 1, pp. 99-112, January, 2014. Article (CrossRef Link)

[27] G. Calinescu, C. Chekuri, M. Pal, and J. Vondrak, "Maximizing a monotone submodular function subject to a matroid constraint," SIAM Journal on Computing, vol. 40. no. 6, pp. 1740-1766, November, 2011. Article (CrossRef Link)

[28] X. Huang, G. Zhao, and Z. Chen, "Segment-based random caching in device-to-device (D2D) caching networks," in Proc. of International Symposium on Wireless Communication Systems, pp. 731-735, August 25-28, 2015. Article (CrossRef Link) 


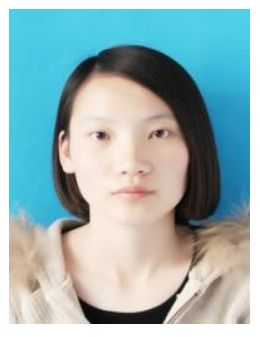

Can Zhang received the B.S. degree from Henan Polytechnic University, Jiaozuo, China in 2016, and the M.S. degree from College of Communications Engineering, Army Engineering University of PLA, Nanjing, China in 2018. Her current research interests include D2D communications, content sharing, content security, and game theory.

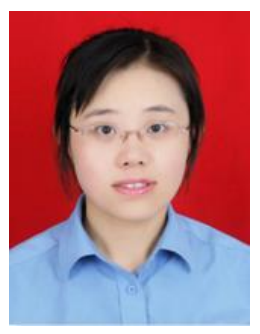

Dan Wu received the B.S., M.S., and Ph.D. degrees from PLAUST, Nanjing, China, in 2006, 2009, and 2012, respectively. She is currently a Postdoctoral Researcher in Army Engineering University of PLA. Her research interests include resource allocation and management, game theory, cooperative communications, and wireless sensor networks.

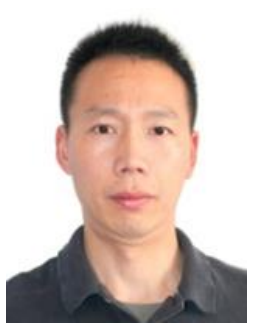

Liang Ao recieved the B.S. and M.S. degrees from PLAUST, Nanjing, China, in 2007, and 2010, respectively. He is currently an Instructor in Army Engineering University of PLA. His research interests include signal and information processing, deep learning, and speech processing.

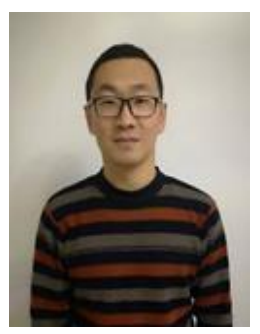

Meng Wang received the B.S. and M.S. degrees in instructional technology from the Nanjing Normal University, Nanjing, China, in 2006 and 2009, respectively. His current interests include wireless network security, D2D communications, and game theory.

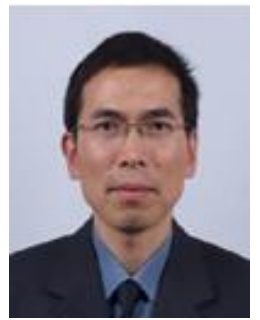

Yueming Cai received his B.S. degree in Physics from Xiamen University, Xiamen, China in 1982, the M.S. degree in Micro-electronics Engineering and the Ph.D. degree in Communications and Information Systems both from Southeast University, Nanjing, China in 1988 and 1996, respectively. His current research interests include MIMO systems, OFDM systems, signal processing in communications, cooperative communications andwireless sensor networks. 